\title{
Sufficient Conditions for Bang-Bang Control in Hilbert Space ${ }^{1}$
}

\author{
E. D. Rogak, ${ }^{2}$ N. D. Kazarinoff, ${ }^{3}$ and J. F. ScotT-Thomas ${ }^{4}$ \\ Communicated by L. Cesari
}

\begin{abstract}
Sufficient conditions for bang-bang and singular optimal control are established in the case of linear operator equations with cost functionals which are the sum of linear and quadratic terms, that is, $A x=u$, $J(u)=(r, x)+\beta(x, x), \beta>0$. For example, if $A$ is a bounded operator with a bounded inverse from a Hilbert space $H$ into itself and the control set $U$ is the unit ball in $H$, then an optimal control is bang-bang (has norm 1 ) if $0 \leqslant \beta<\frac{1}{2}\left\|A^{-1 *} r\right\| \cdot\left\|A^{-1}\right\|^{-2}$, but is singular (an interior point of $U$ ) if $\beta>\frac{1}{2}\left\|A^{-1 *} r\right\| \cdot\|A\|^{2}$.
\end{abstract}

\section{Introduction}

Control processes described by differential equations linear in the control but with quadratic cost functionals are often not bang-bang (Refs. 1-3). That is, for such processes, the optimal control does not lie on the boundary of the control region. A simple example of such a process is described in Section 2, namely, to minimize

$$
J(u)=\beta \int_{0}^{T} x^{2} d t, \quad \beta>0
$$

for $u$ piecewise constant and $|u(t)| \leqslant 1$ on $[0, T]$ if

$$
d x / d t=u, \quad x(0)=x_{0}, \quad x(T)=x_{1}
$$

${ }^{1}$ Paper received July 24, 1969. This work was supported by NRC Grant No. A-4047 and NSF Grant No. GP-7445.

2 Assistant Professor, Department of Mathematics, University of Victoria, Victoria, British Columbia, Canada.

'Professor, Department of Mathematics, University of Michigan, Ann Arbor, Michigan.

"Assistant Professor, Department of Mathematics, University of Victoria, Victoria, British Columbia, Canada.

(C) 1970 Plenum Publishing Corporation, New York, N.Y. 
Here, $x_{0}, x_{1}, T$ are fixed. Whether or not a given finite-dimensional process is bang-bang can be determined from Pontryagin's maximum principle, provided the adjoint problem can be solved and has a nonzero solution. However, a maximum principle for control problems involving operator equations in a Hilbert space is not presently available except in special cases (Refs. 4-5).

Here, we establish, without a maximum principle, sufficient conditions for bang-bang and singular optimal control (in the sense defined in Section 3) in the case of linear operator equations with cost functionals which are the sum of linear and quadratic terms, that is,

$$
A x=u, \quad J(u)=(r, x)+\beta(x, x), \quad \beta>0
$$

These are stated as Theorems 3.1, 4.1, 4.2 in Sections 3 and 4. We show, for example, that, if $A$ is a bounded operator with a bounded inverse from a Hilbert space $H$ into itself and the control set $U$ is the unit ball in $H$, then an optimal control is bang-bang (has norm 1) if

$$
0 \leqslant \beta<\frac{1}{2}\left\|A^{-1^{*}} r\right\| \cdot\left\|A^{-1}\right\|^{-2}
$$

but is singular (an interior point of $U$ ) if

$$
\beta>\frac{1}{2}\left\|A^{-1^{*}} r\right\| \cdot\|A\|^{2}
$$

We were led to our results through consideration of the example mentioned above. Our approach to guarantee bang-bang control is the naive one: we assume that the optimal control $\tilde{u}$ is singular and, with simple estimates, show that there exists a $\lambda>0$ such that $J(\lambda \tilde{u})<J(\tilde{u})$. In essence, our results are: if $J$ is close to linear, an optimal control is bang-bang; but, if $J$ is close to quadratic, the optimal control is singular. They appear to be reasonably close to best possible.

\section{Finite-Dimensional Example}

Consider the problem represented by

$$
d x / d t=u, \quad x(0)=x_{0}, \quad x(T)=x_{1}
$$

with

$$
J(u)=\int_{0}^{T}\left(\beta x^{2}+r x\right) d t, \quad \beta>0
$$




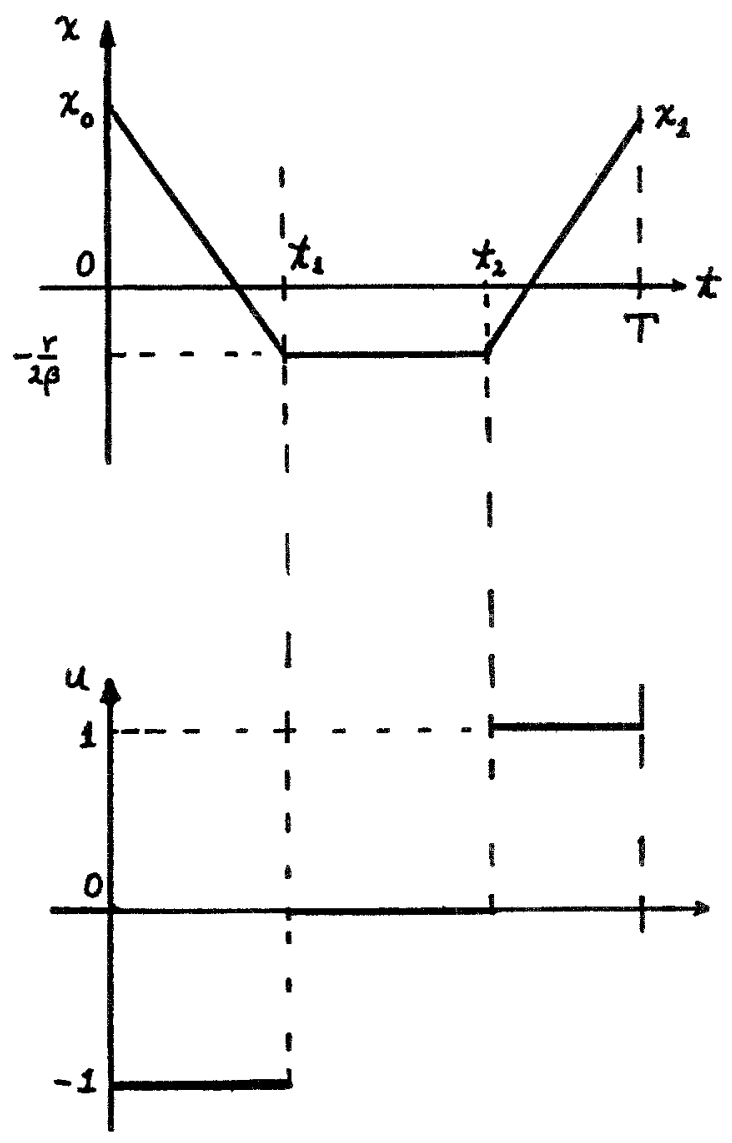

Fig. 1

Here, $x_{0}, x_{1}, T$ are fixed; $r, \beta$ are real numbers; and $u \in U=\{u \mid u$ is piecewise constant and $|u(t)| \leqslant 1$ on $[0, T]\}$. The problem is to minimize $J(u)$ for $x(t, u)$ satisfying $(1)$ and $u \in U$. We assume that $x_{1}$ is accessible from $x_{0}$ in the given time $T$.

The minimum of $\beta x^{2}+r x$ occurs at $x=-r / 2 \beta$. Optimum passage from $x_{0}$ to $x_{1}$ in time $T$ is obtained by moving as fast as possible to $x=-r / 2 \beta$ (with $u=-1$ if $x_{0}>-r / 2 \beta$, but with $u=+1$ if $x_{0}<-r / 2 \beta$ ). The process continues at $x=-r / 2 \beta(u \equiv 0)$ until we can apply a control $u= \pm 1$, the sign depending upon whether $x_{1}$ is larger or smaller than $-r / 2 \beta$, to reach $x_{1}$ at time $T$. The switching times are

$$
t_{1}=x_{0}+(r / 2 \beta), \quad t_{2}=T-\left[x_{1}+(r / 2 \beta)\right]
$$


The phase trajectory and control are shown for a typical case $\left(x_{0}\right.$ and $x_{1}$ exceed $-r / 2 \beta)$ in Fig. 1. Thus, if both $x_{0}$ and $x_{1}$ exceed $-r / 2 \beta$, a necessary and sufficient condition for bang-bang control is $t_{2} \leqslant t_{1}$, that is,

$$
r / \beta \geqslant T-\left(x_{0}+x_{1}\right)
$$

Analogous results hold for other configurations of $x_{0}$ and $x_{1}$.

The reader should be aware that, while the example just described motivated the discovery of the theorems to follow, we have been unable to fit the example in the abstract setting of the theorems. Our difficulty is that some nonextremal points of the control set in the example are identified as extremal points when we try to describe the example in terms of the abstract setting of the theorems.

\section{Bang-Bang Control}

Let $H_{i}, i=1,2$, be two real Hilbert spaces with inner products and norms $(.,)_{i}$ and $\|\cdot\|_{i}$, respectively. We assume that $A$ is a linear operator from $H_{1}$ into $H_{2}$, that is,

$$
A: \quad \mathscr{D}(A) \stackrel{1-1}{\leftrightarrow} R(A)
$$

with $R(A)$ dense in $H_{2}$. For the control region $U$, we choose a subset of $R(A)$. In all that follows, it is sufficient to assume that $A$ has a right inverse and that $R(A)$ is dense in a subspace of $H_{2}$. We have made the stronger and simpler assumptions that $A$ has an inverse and $R(A)$ is dense in $H_{2}$.

Let $r \in \mathscr{D}\left(A^{-1 *}\right)$ be a fixed vector in $H_{1}$. We study controls $u$ that minimize the functional

$$
J(u)=(r, x)_{1}+\beta(x, x)_{1}, \quad \beta \geqslant 0
$$

subject to the constraint

$$
A x=u
$$

We assume that an optimal control exists; namely, for all $\beta \geqslant 0$, there exists at least one $\tilde{u} \in U$ and $\tilde{x} \in \mathscr{D}(A)$ such that

$$
A \tilde{x}=\tilde{u} \quad \text { and } \quad J(\tilde{u})=\inf _{u \in U} J(u)
$$

Note that $\tilde{u}$ and $\tilde{x}$ in general depend on $\beta$. Also, we need not assume uniqueness of the optimal control. However, it turns out that, if $U$ is convex, then the 
optimal control must be unique. In fact, let $u_{0}$ and $u_{1}$ be two optimal controls corresponding to $x_{0}$ and $x_{1}$, with $J\left(u_{0}\right)=J\left(u_{1}\right)$. Then, $(1-\lambda) u_{0}+\lambda u_{1}$ is an admissible control corresponding to $(1-\lambda) x_{0}+\lambda x_{1}$. A simple computation shows that

$$
\begin{aligned}
J\left(x_{\lambda}\right) & =(1-\lambda) J\left(u_{0}\right)+\lambda J\left(u_{1}\right)-\lambda(1-\lambda) \beta\left(x_{0}-x_{1}, x_{0}-x_{1}\right) \\
& <J\left(x_{0}\right) \quad \text { if } \quad u_{0} \neq u_{1}
\end{aligned}
$$

Further, it is natural to impose the condition that, even when $\beta=0$, the control action affects $J$; namely, if $\beta=0$, there exists a $v \in U$ such that

$$
J(v)=\left(r, A^{-1} v\right)_{1}+O\left(A^{-1} v, A^{-1} v\right)_{1}=\left(A^{-1^{*}} r, v\right)_{2}=\theta \neq 0
$$

In what follows, $v$ is an optimal control for $\beta=0$.

Note that, if $U$ is a subset of the unit ball and

$$
-r^{*} /\left\|r^{*}\right\| \in U \quad\left(r^{*}=A^{-1^{*}} r\right)
$$

then it is an optimal control for $\beta=0$, and the minimum value of $J$ is $-\left\|r^{*}\right\|_{2}$. In the case $\beta=0$, whether or not $-r^{*} /\left\|r^{*}\right\| \in U$, the optimal control process is bang-bang. On the other hand, if $r=0$, then $J(u)=\beta(x, x)$ is least for $u=x=0$. In this case, therefore, the optimal control is singular. The theorems to follow give various sufficient conditions to guarantee bang-bang control or singular control in the intermediate cases where neither $r$ nor $\beta$ is $z$ ero.

We next describe the kind of control sets we shall admit, and define precisely what we mean by bang-bang control.

Definition 3.1. A control set $U$ is star-shaped if, and only if, for each $u \in U$, there exists an $\epsilon>0$ such that $\lambda u \in U$ for $-\epsilon<\lambda<1$.

Definition 3.2. A point $u$ in a control set $U$ is an extremal point of $U$ if, and only if, $\lambda u \notin U$ for each $\lambda>1$.

A star-shaped control set, according to Definition 3.1 , may be a thin spiny set or one with "faces" containing "radial line segments". Hence, not all the boundary points of a star-shaped control set are necessarily extremal points. By a bang-bang control, we mean an extremal point of $U$. By a singular control, we mean one that is not bang-bang. A singular control may be a boundary point of $U$.

It is easy to see that extremal points are always boundary points and, if $U$ is a convex body with the origin in its interior, then it is also star-shaped. 
Theorem 3.1. If $U$ is a star-shaped subset of the unit ball of $H_{2}$ and if

$$
A: \quad \mathscr{D}(A) \stackrel{1-1}{\leftrightarrow} R(A) \subset H_{2}
$$

has a bounded inverse. with $\left\|A^{-1}\right\|^{2}=K$, then an optimal control for the problem (3)-(4) is always an extremal point of $U$ for all nonnegative

$$
\beta<\|\theta\| / 2 K
$$

where $\theta=\min _{u \in U} J(u)$ for $\beta=0$.

Note that $A$ need not be bounded, nor must $\tilde{u}$ be unique.

Proof. Suppose that $\tilde{u}$, an optimal control, is not an extremal point of $U$. Then, since $U$ is star-shaped, there exists an $\epsilon_{0}>0$ such that, if $1 \leqslant \lambda<1+\epsilon_{0}, \lambda \tilde{u} \in U$. Thus, we may consider

$$
J(\lambda \tilde{u})-J(\tilde{u})=(\lambda-1)\left[\left(r^{*}, \tilde{u}\right)_{2}+\beta\left(L_{1} \tilde{u}, \tilde{u}\right)_{2}+\lambda \beta\left(L_{1} \tilde{u}, \tilde{u}\right)_{2}\right]
$$

where

$$
r^{*}=A^{-1^{*}} r \quad \text { and } \quad L_{1}=A^{-1^{*}} A^{-1}
$$

By (6), there exists an $\epsilon>0$ such that

$$
\beta<|\theta| /(2+\epsilon) K<|\theta| / 2 K
$$

Therefore, recalling the definition of $v$ just after (5), we obtain

$$
\begin{aligned}
\left(r^{*}, \tilde{u}\right)_{2}+\beta\left(L_{1} \tilde{u}, \tilde{u}\right)_{2} & \equiv J(\tilde{u}) \leqslant J(v) \equiv\left(r^{*}, v\right)_{2}+\beta\left(L_{1} v, v\right)_{2} \\
& \leqslant \theta+\beta\left|\left(L_{1} v, v\right)_{2}\right| \leqslant \theta+\beta K<\theta+|\theta| /(2+\epsilon) \\
& =(1+\epsilon) \theta /(2+\epsilon)<0
\end{aligned}
$$

The last equality holds, since the hypotheses that $U$ is star-shaped and the control action affects $J$ if $\beta=0$ guarantee that $\theta<0$.

We next estimate the remaining term in (7). Suppose that $\epsilon_{0}<\epsilon / 2$. Then,

$$
\left|\lambda \beta\left(L_{1} \tilde{u}, \tilde{u}\right)_{2}\right|<\lambda|\theta| /(2+\epsilon)<(1+\epsilon / 2)|\theta| /(2+\epsilon)=-\theta / 2
$$

Thus,

$$
\begin{aligned}
J(\lambda \tilde{u})-J(\tilde{u}) & <(\lambda-1)\{[(1+\epsilon) \theta /(2+\epsilon)]-(\theta / 2)\} \\
& \leqslant(\epsilon / 2) \epsilon \theta / 2(2+\epsilon)<0
\end{aligned}
$$

This contradicts the optimality of $\tilde{u}$. 
Corollary 3.1. If, in addition to the hypotheses of the theorem,

$$
r^{*} /\left\|r^{*}\right\| \in U
$$

then $\tilde{u}$ is always an extremal point of $U$ for all nonnegative

$$
\beta<\left\|r^{*}\right\|_{2} / 2\left\|A^{-1}\right\|^{2}
$$

Recall that a regular convex body is one in which every hyperplane of support intersects the body in exactly one point. An extreme point of a convex set $U$ is one that is not a nontrivial convex combination of other points in $U$.

Corollary 3.2. Let the properties of $A$ be as in Theorem 3.1. Let $U$ be a regular convex body contained in the unit ball and containing the origin. If

$$
\beta<|\theta| / 2 K
$$

then the unique optimal control $\tilde{u}$ is an extreme point of $U$.

Proof. The uniqueness follows from the convexity of $U$ and the remarks preceding (5). Now, suppose that there existed $y, w \in U$, and $0<t<1$ such that $\tilde{u}=t y+(1-t) w$. By Theorem 3.1, $\tilde{u}$ is extremal and, hence, for all $\lambda>1, \lambda \tilde{u} \notin U$. We now show that the segment $[y, w]$ is composed only of extremal points. For $\alpha>1$, if $\alpha y \in U$, then the segment $[w, \alpha y] \subset U$. But this segment cuts the segment $[0, \lambda \tilde{u}]$ at some point $\tilde{\lambda} \tilde{u}, \delta>1$, contradicting the extremality of $\tilde{u}$. Thus, $y$ and, similarly, $w$ are extremal points of $U$. The same argument shows that all points in the segment $[y, w]$ must be extremal and, hence, boundary points of $U$. By a well-known theorem (Ref. 6, p. 64), there exists a closed hyperplane separating $[y, w]$ and int $U$, the interior of $U$. It is easy to see that this must be a hyperplane of support of $U$, containing $[y, w]$, and thus contradicting the regular convexity of $U$.

\section{Singular Control}

For $A$ unbounded and $A^{-1}$ bounded, we have not been able to prove, as we would have liked to have done, that an optimal control for the problem (4)-(5) is never an extremal point of $U$ if

$$
\beta>|\theta| / 2 K
$$

In the way of an only if part of Theorem 3.1 we have only been able to prove 
that optimal controls are singular if $\beta$ is sufficiently large. How large we do not know. better.

Under alternate hypotheses to those of Theorem 3.1, however, we can do

Theorem 4.1. If (a) $U$ is a star-shaped subset of the unit ball in $H_{2}$, (b) $A: \mathscr{D}(A) \stackrel{1,-1}{\leftrightarrow} R(A) \subset H_{2}$ is a bounded linear operator from $H_{1}$ to $H_{2}$, with $A^{-1}$ bounded or unbounded, and $(c)$

$$
\inf \{\|u\| \mid u \in U \text { and } u \text { is extremal }\}=m>0
$$

then an optimal control $\tilde{u}$ for the problem (4)-(5) is never an extremal point (is always singular) if

$$
\beta>\left\|r^{*}\right\|_{2}\|A\|^{2} / 2 m^{2}
$$

Proof. Suppose that $\beta$ satisfies (9) and $\tilde{u}$, an optimal control, is an extremal point of $U$. Since $U$ is star-shaped, $\lambda \tilde{u} \in U$ for all $\lambda \in[0,1]$. For such $\lambda$, we may consider

$$
J(\lambda \tilde{u})-J(\tilde{u})=(\lambda-1)\left[\left(r^{*}, \tilde{u}\right)_{2}+(\lambda+1) \beta\left(L_{2} \tilde{u}, \tilde{u}\right)_{2}\right]
$$

where

$$
r^{*}=A^{-1^{*}} r \quad \text { and } \quad L_{1}=A^{-1^{*}} A^{-1}
$$

Now,

$$
\left|\left(r^{*}, \tilde{u}\right)_{2}\right| \leqslant\left\|r^{*}\right\|_{2}
$$

since $\|\tilde{u}\| \leqslant 1$. Also, by the hypothesis (8),

$$
m \leqslant\|\tilde{u}\|_{2}=\|A \tilde{x}\|_{2} \leqslant\|A\|\|\tilde{x}\|_{1} \quad(A \tilde{x}=\tilde{u})
$$

or

$$
\|\tilde{x}\|_{1}^{2} \geqslant m^{2} /\|A\|^{2}
$$

Therefore,

$$
\left(L_{1} \tilde{u}, \tilde{u}\right)_{2}=\|\tilde{x}\|_{1}^{2} \geqslant m^{2} /\|A\|^{2}
$$

It now follows from (10)-(11) that

$$
\begin{aligned}
\left(r^{*}, \tilde{u}\right)_{2}+(\lambda+1) \beta\left(L_{1} \tilde{u}, \tilde{u}\right)_{2} & \geqslant-\|\left(r^{*}, \tilde{u}\right)_{2} \mid+\beta(\lambda+1)\left(L_{1} \tilde{u}, \tilde{u}\right)_{2} \\
& \geqslant-\left\|r^{*}\right\|_{2}+\beta(\lambda+1)\left[m^{2} /\|A\|^{2}\right]
\end{aligned}
$$


But, for $\beta$ satisfying (9), there exists an $\epsilon \in(0,1)$ such that

$$
\beta>\left\|r^{*}\right\| \cdot\|A\|^{2} /(\epsilon+1) m^{2}
$$

Having chosen $\epsilon \in(0,1)$, we finally choose $\lambda \in(\epsilon, 1)$. Then, by (12)-(13),

$$
\begin{aligned}
\frac{J(\lambda \tilde{u})-J(\tilde{u})}{\lambda-1} & >-\left\|r^{*}\right\|_{2}+\frac{\left\|r^{*}\right\|_{2}\|A\|^{2}}{(\epsilon+1) m^{2}}(\lambda+1) \frac{m^{2}}{\|A\|^{2}} \\
& =\left\|r^{*}\right\|_{2}\left[-1+\frac{1+\lambda}{1+\epsilon}\right]>0
\end{aligned}
$$

Since $\lambda-1<0$, we now are forced to conclude that

$$
J(\lambda \tilde{u})-J(\tilde{u})<0
$$

This contradicts the optimality of $\tilde{u}$.

Corollary 4.1. If (a) $U$ is the unit ball in $H_{2}$, (b) $A$ is a bounded linear operator from $H_{1} \rightarrow H_{2}$ with a bounded inverse, and (c)

$$
\|A\|^{2}=\left\|A^{-1}\right\|^{-2}=K
$$

then an optimal control $\tilde{u}$ for the problem (4)-(5) is bang-bang if

$$
0 \leqslant \beta<\frac{1}{2}\left\|r^{*}\right\|_{2} K
$$

and is singular if

$$
\beta>\frac{1}{2}\left\|r^{*}\right\|_{2} K
$$

Example 4.1. Suppose that $H_{1}=H_{2}=R^{2}$, the plane, and suppose that $U$ is the closed unit disc. Consider

$$
r=\left[\begin{array}{l}
1 \\
0
\end{array}\right]
$$

and assume $A$ to be a rotation of $R^{2}$ about the origin counterclockwise through an angle $\theta$. Then, $\|A\|=\left\|A^{-1}\right\|^{-1}=1$, and

$$
J(u)=(\cos \theta): u_{1}+(\sin \theta) u_{2}+\beta\left(u_{1}^{2}+u_{2}^{2}\right)
$$
for

For $\beta>0$, the minimum value of $J(u)$ over all of $R^{2}$ is $-1 / 4 \beta$ and is taken

$$
u=\left[\begin{array}{l}
u_{1} \\
u
\end{array}\right]=\left[\begin{array}{l}
-\cos \theta / 2 \beta \\
-\sin \theta / 2 \beta
\end{array}\right]
$$


It is easy to see geometrically that, if $0<\beta \leqslant \frac{1}{2}$, the minimum of $J(u)$ for $u$ in the closed unit disc $U$ is $-1+\beta$ and is taken on for

$$
u=\left[\begin{array}{rr}
\cos \theta & -\sin \theta \\
\sin \theta & \cos \theta
\end{array}\right]\left[\begin{array}{r}
-1 \\
0
\end{array}\right]=\left[\begin{array}{l}
-\cos \theta \\
-\sin \theta
\end{array}\right]
$$

(If $0<\beta<\frac{1}{2}, \min J(u)$ over $R^{2}$ is attained outside $U$.) This control is bangbang.

For $\beta \geqslant \frac{1}{2}$, as $\beta \nearrow+\infty$, the minimum of $J(u)$ on $U$ is its minimum over all of $R^{2}$, namely, $-1 / 4 \beta$, which is taken at the point given by (14). Thus, as $\beta \nearrow \infty, \tilde{u}$ approaches 0 along the radius with endpoint $(-\cos \theta,-\sin \theta)$. This control is singular. For this example, Theorems 3.1 and 4.1 are the best possible.

Finally, we give the only if part of Theorem 3.1.

Theorem 4.2. If (a) $U$ is a subset of the unit ball in $H_{2}$, (b) the set

$$
\mathscr{E}=\{u \mid u \in U \text { and } u \text { is extremal }\}
$$

is compact, (c) there exists an $\epsilon>0$ such that, if $u \in \mathscr{E},\|u\| \geqslant \epsilon$, and (d) $A$ is a (not necessarily bounded) linear operator from $H_{1}$ into $H_{2}$ with a bounded inverse, then an optimal control $\tilde{u}$ for the problem (4)-(5) is always singular for $\beta$ sufficiently large.

Proof. Suppose that the theorem is false. Then, there exist $\beta_{n} \nearrow \infty$ such that, to each $\beta_{n}$, there corresponds an optimal control $\tilde{u}_{n}$ with $\tilde{u}_{n} \in \mathscr{E}$. Since $J(0)=0$ for all $\beta_{n}$,

$$
J\left(\tilde{u}_{n}\right) \equiv\left(r^{*}, \tilde{u}_{n}\right)_{2}+\beta_{n}\left(L_{1} \tilde{u}_{n}, \tilde{u}_{n}\right)_{2} \leqslant 0
$$

But

$$
\left(L_{\mathbf{1}} \tilde{u}_{n}, \tilde{u}_{n}\right)=\left\|A^{-1} \tilde{u}_{n}\right\|^{2} \leqslant\left\|A^{-1}\right\|^{2}\left\|u_{n}\right\|^{2}<\infty
$$

Therefore, it follows from (15) that, since $\beta_{n} \nearrow \infty$, either

$$
\left(r^{*}, \tilde{u}_{n}\right) \rightarrow-\infty
$$

(which is impossible) or

$$
\left\|A^{-1} \tilde{u}_{n}\right\| \rightarrow 0
$$

In the latter case, since $\mathscr{E}$ is compact, there exists a convergent subsequence of $\left\{\tilde{u}_{n}\right\}$, which we also call $\left\{\tilde{u}_{n}\right\}$, such that

$$
\tilde{u}_{n} \underset{n \rightarrow \infty}{\longrightarrow} \tilde{u} \in \mathscr{E} \quad \text { in } \quad H_{2}
$$


Since $A^{-1}$ is bounded,

$$
A^{-1} \tilde{u}_{n} \underset{n \rightarrow \infty}{\longrightarrow} A^{-1} \tilde{u} \quad \text { in } \quad H_{1}
$$

The alternative (16) thus implies that $\tilde{x}=A^{-1} \tilde{u}=0$ and, in turn, $\tilde{u}=0$. This contradicts hypothesis (C) of the theorem.

\section{References}

1. Feldbaum, A. A., Optimal Control Systems, Academic Press, New York, 1965.

2. Rozonoer, L. I., Pontryagin's Maximum Principle in the Theory of Optimum Systems, Automation and Remote Control, Vol. 20, No. 10, 1959.

3. Wonham, W., and Johnson, C. D., Optimal Bang-Bang Control with Quadratic Performance Index, ASME Transactions, Series D, Vol. 86, No. 1, 1964.

4. Egorov, A. I., Optimal Control in a Banach Space, Mathematical Systems Theory, Vol. 1, No. 4, 1967.

5. EGorov, YU., Necessary Conditions for Optimality in Banach Space, Matematicheskii Sbornik, Vol. 1, No. 4, 1969.

6. Schaefer, H., Topological Vector Spaces, The Macmillan Company, New York, 1966. 